in dem neuen Patentgesetz in $\$ 1$, Abs. 2, Z. 2 die Worte, ,sowie von Stoffen, welche auf chemischem Wege hergestellt werden" zu streichen, ev. sodann in $\$ 4$ folgende Restimmung einzufügen:

..Ist der Gegenstand der Erfindung ein neuer chemischer Stoff, so hat das Patent keine Wirkung gegen später angemeldete Patente, welche ein neues Verfahren zur Herstellung dieses Stoffes betreffen."

[A. 140.]

\section{Zur \\ Theorie des Bleikammerprozesses.}

\author{
Von O. W $_{\text {ENTZKI }}{ }^{1}$ ).
}

(Eingeg. 8./8. 1910.)

Trotzdem seit mehr als 150 Jahren Schwefelsäure nach dem Bleikammerverfahren hergestellt wird, ist die Frage nach den bei diesem Prozesse sich abspielenden chemischen Vorgängen bis auf den heutigen Tag noch unerledigt, $d$. h. es liegt eine allseitig befriedigende Erklärung über die Wirkung der Stickstoffoxyde in den Bleikammern noch nicht vor. Ja, beim Durchlesen der einschlägigen neueren Literatur kann man sogar zu der Auffassung gelangen, daß wir von der Lösung des Problems weiter entfernt sind, wie vor 100 Jahren.

Die Erkenntnis der-Vorgänge in den Bleikammern wird hauptsächlich erschwert durch die große Geschwindigkeit, mit der die Oxydation des Schwefeldioxyds erfolgt oder vielmehr durch die hierdurch bedingte Unmöglichkeit, den Hauptprozeß meßbar zu verfolgen; es wirken aber auch die Nebenreaktionen störend.

Bei der Ausarbeitung eines neuen Verfahrens zur Darstellung von Schwefelsäure habe ich einige Beobachtungen gemacht, die ich hier mitteilen möchte, weil sich daraus Schlüsse auf den Reaktionsverlauf in der Bleikammer und auf die Richtigkeit gewisser Theorien des Bleikammerprozesses ziehen lassen.

Bevor ich auf die betreffenden Versuche eingehe, möge mir gestattet sein, kurz einige der bekanntesten Theorien des Bleikammerprozesses zu rekapitulieren. um meine weiteren Ausführungen daran anknüpfen zu können.

Nach einer allgemeineren Annahme vollzieht sich die Oxydation des Schwefeldioxyds zu Schwefelsäure über die Nitrosylschwefelsäure, wie dies bereits Davy behauptet hat. Nach seiner Auffassung spielt sich der Bleikammerprozeß nach folgenden Gleichungen ab:

(1) $2 \mathrm{SO}_{2}+3 \mathrm{NO}_{2}+\mathrm{H}_{2} \mathrm{O}+2 \mathrm{SO}_{2}(\mathrm{OH}) \mathrm{ONO}+\mathrm{NO}$

(2) $2 \mathrm{SO}_{2}(\mathrm{OH}) \mathrm{ONO}+\mathrm{H}_{2} \mathrm{O}$

(3) $2 \mathrm{NO}+2 \mathrm{O}=2 \mathrm{NO}_{2}$.

$$
=2 \mathrm{SO}_{2}(\mathrm{OH})_{2}+\mathrm{NO}_{2}+\mathrm{NO}
$$

Diese Theorie stützt sich auf die von $D$ a $v$ y beobachtete Tatsache, daß $\mathrm{SO}_{2}$ und $\mathrm{NO}_{2}$, trocken zusammengebracht, nicht miteinander reagieren, wohl aber bei Gegenwart von Wasser unter Bildung von Schwefelsäure. Ist die Menge des Wassers gering, so entstehen die bereits von $\mathrm{Cl}$ e men $\mathrm{t}$ und Des or mes beobächteten Bleikammerkrystalle,

1) Vortrag gehalten im Frankfurter Bezirksverein in der Sitzung vom 4./6. 1910. d. i. Nitrosylschwefelsäure. Da letztere mit Wasser in Schwefelsäure und salpetrige Säure ( $\left.\mathrm{NO}+\mathrm{NO}_{2}\right)$ zerfällt, so hielt $\mathrm{Davy}$ die Bildung der Nitrosylschwefelsäure aus $\mathrm{SO}_{2}, \mathrm{NO}_{2}$ und $\mathrm{H}_{2} \mathrm{O}$ resp. den Zerfall derselben durch $\mathrm{Hydrolyse}$ in $\mathrm{H}_{2} \mathrm{SO}_{4}$ und $\mathrm{N}_{2} \mathrm{O}_{3}$ beim BleikammerprozeB für erwiesen.

Daß die Nitrosylschwefelsäure hier als notwendige Zwischenstufe anzusehen sei, darin stimmen viele Chemiker, die sich mit dem Studium des Prozesses beschäftigt haben, mit $D$ a v y überein, jedoch gehen die Ansichten hinsichtlich der Art der Bildung der Nitrosylschwefelsäure vielfach und ganz wesentlich auseinander. Während z. B. Cl. W i n $\mathrm{k}$ l e r dabei dem Stickstoffdioxyd die Hauptrolle zuweist und analog der $\mathrm{D}$ a $v$ y schen Theorie einen Reduktions- und einen Oxydationsvorgang annimmt, ist nach der im Jahre 1885 von $\mathrm{L} u \mathbf{u} \mathrm{g} \mathrm{e}^{2}$ ) aufgestellten Theorie das Stickstofftrioxyd als Sauerstoffüberträger anzusehen.

Die Bildung der Schwefelsäure ist nach $\mathrm{L}$ u $\mathrm{n}$ g e s damaliger Anschauung, die auch heute noch von vielen Chemikern geteilt wird, nicht auf eine abwechselnde Oxydation des Stickstoffoxyds zu Stickstofftrioxyd oder Stickstofftetraoxyd und darauffolgende Reduktion dieser Stickstoffox yde zu NO zurückzuführen, dieselbe erfolgt vielmehr dadurch, daß zunächst eine Kondensation von Stickstofftrioxyd, Schwefeldioxyd, Sauerstoff und Wasser erfolgt und hierauf durch Hydrolyse eine Spaltung der gebildeten Nitrosylschwefelsüure in Schwefelsäure und Stickstofftrioxyd eintritt entsprechend den Gleichungen

(4) $2 \mathrm{SO}_{2}+\mathrm{NO}+\mathrm{NO}_{2}+\mathrm{O}_{2}+\mathrm{H}_{2} \mathrm{O}=2 \mathrm{NO}_{2} \mathrm{SO}_{3} \mathrm{H}$

(5) $2 \mathrm{SO}_{3} \mathrm{HNO}_{2}+\mathrm{H}_{2} \mathrm{O}=2 \mathrm{H}_{2} \mathrm{SO}_{4}+\mathrm{NO}+\mathrm{NO}_{2}$.

L u $\mathrm{ng}$ e drückt dies in folgender Weise aus: „Das Schwefeldioxyd tritt unmittelbar mit Stickstofftrioxyd, Sauerstoff und wenig Wasser zu Nitrosylschwefelsäure zusammen, welche nebelförmig in der Kammer schwebt; beim Zusammentreffen mit mehr Wasser, welches ebenfalls als Nebel in der Kammer verteilt ist, zerlegt sich die Nitrosylschwefelsäure in Schwefelsäure, die zu Boden sinkt, und Stickstofftrioxyd, welches von neuem wirken kann. Es ist also nicht, wie die bisher allgemein herrschende Ansicht lautet, das Stickoxyd, sondern das Salpetrigsëureanhydrid, welches als Sauerstoffüberträger in der Bleikammer fungiert."

Außer den Reaktionen (4) und (5) könnea nach L u $\mathrm{n}$ g es Ansicht (loc. cit.) noch folgende stattfinden:

(6) $\mathrm{SO}_{2}+\mathrm{NO}_{2}+\mathrm{H}_{2} \mathrm{O}=\mathrm{H}_{2} \mathrm{SO}_{4}+\mathrm{NO}$,

(7) $\mathrm{SO}_{2}+\mathrm{N}_{2} \mathrm{O}_{3}+\mathrm{H}_{2} \mathrm{O}=\mathrm{H}_{2} \mathrm{SO}_{4}+2 \mathrm{NO}$,

(8) $2 \mathrm{SO}_{2}+2 \mathrm{NO}+. \mathrm{O}_{3}+\mathrm{H}_{2} \mathrm{O}=2 \mathrm{SO}_{5} \mathrm{NH}$,

(9) $\mathrm{SO}_{2}+\mathrm{HNO}_{3}=\mathrm{SO}_{5} \mathrm{NH}$,

$(10) 2 \mathrm{SO}_{2}+\mathrm{N}_{2} \mathrm{O}_{4}+\mathrm{O}+\mathrm{H}_{2} \mathrm{O}=2 \mathrm{SO}_{5} \mathrm{NH}$,

(11) $2 \mathrm{SO}_{5} \mathrm{NH}+\mathrm{SO}_{2}+2 \mathrm{H}_{2} \mathrm{O}=3 \mathrm{SO}_{4} \mathrm{H}_{2}+2 \mathrm{NO}$.

Von diesen Reaktionen schreibt. L u n ge jedoch nur der Reaktion 11 eine wesentliche Rolle zu. Die Reaktion spielt sich nach seiner Ansicht vorwiegend im ersten Teile des Kammersystems ab, wodurch auch das Vorhandensein großer Mengen von Stickoxyd daselbst zu erklären ist.

2) Handb. der Schwefelsäurefabrikation 1903, S. 671 . 
Neuerdings hat $\mathrm{L}$ u n g e seine im Jahre 1885 veröffentlichte Theorie wesentlich modifiziert. Wio T ra u tz und R a s chig vor ihm, zog er im Anschlusse an eine längere mit $R$ a $s \mathrm{ch}$ i g geführte Kontroverse in einer gemeinschaftlich mit $\mathrm{B}$ e r l veröffentlichten Arbeit ${ }^{3}$ ) zur Erklärung der Vorgänge in den Bleikammern die Sulfonitronsäure $\left(\mathrm{H}_{2} \mathrm{SNO}_{5}\right)$ oder, wio $\mathrm{R}$ a s c hi g sie nennt, die $\mathrm{Ni}$ trosisulfosäure als weitere $Z$ wischenstufe heran.

Da die Sulfonitronsäure in der Theorie von L u n g e, B e r l und R a s c hig eine große Rolle spielt, und sie außerdem ein sehr interessanter Körper ist, so möchte ich etwas näher auf denselben eingehen. Man kann ihn nach verschiedenen Methoden erhalten, z. B. durch Einwirkung von Schwefeldioxyd auf nitrose Säure. Ich benutzo dabei einen ca. $60 \mathrm{~cm}$ langen und $6 \mathrm{~cm}$ weiten Glaszylinder (s. Abb.), der an beiden Enden zugeschmol-

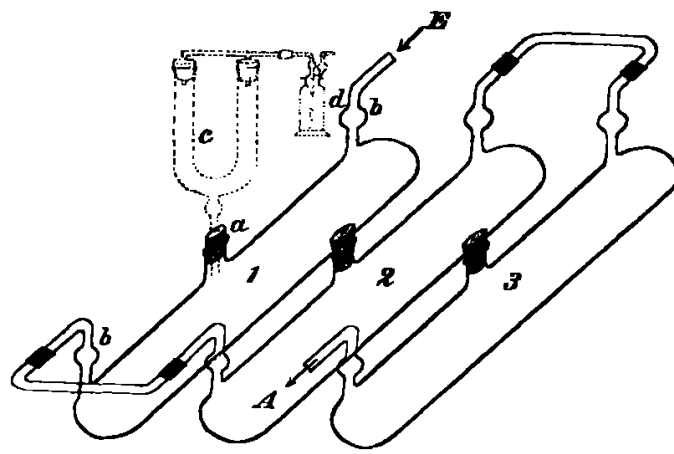

zen ist. Auf der Oberseite des Zylinders4) befinden sich in der Mitte ein Füllstutzen a und in der Nähe der Enden zwei Kugelröhren b, die zum Zu- und Ableiten der Reaktionsyase dienen. Bei der Darstellung der blauen Verbindung (Sulfonitronsäure) fülle ich den Zylinder zu drei Vierteln mit nitroser Säure vom spez. Gew. 1,70 - hergestellt durch Auflösen von $20 \mathrm{~g}$ Natriumnitrit in $1000 \mathrm{~g}$ 60grädiger Schwefelsäure - und leite dann Schwefeldioxyd durch den Apparat. Wird hierbei durch senkrecht zur Achse des Zylinders ausgeführte Schüttelbewegungen die nitrose Säure in innige Berührung mit dem Schwefeldioxyd gebracht, so färbt sich die Säure sehr bald blauviolett. Die Färbung tritt zunëchst im vorderen Teile des Apparates auf und schreitet allmählich nach hinten fort. Gleichzeitig mit der Färbung bemerkt man in der Flüssigkeit die Entwicklung zahlreicher Bläschen von Stickstoffoxyd. Wenn man jetzt den Schwefeldioxydstrom abstellt und Luft durch den Zylinder leitet, so verschwindet die Blaufärbung und hört auch die Entwicklung von Stickoxyd, auf. Denselben Effekt kann man auch durch Zusatz von einigen Tropfen Salpetersäure erzielen.

Diese blaue Verbindung, von den Engländern Purple acid genannt, ist beim Bleikammerbetrieb ofters beobachtet worden. Sie hat schon viele Forscher beschäftigt. Alle stimmen darin überein, daß der Körper Säurecharakter besitzt, weil er mit gewissen Metallen salzähnliche Verbindungen eingeht,

3) Diese Z. 19, 886 (1906).

4) Derartige Zylinder sowie der weiter unten beschriebene Apparat zur Darstellung von Schwefelsäure liefert die Firma Dr. Bachfeld \& Co. in Frankfurt.a. $M$. die allerdings seither nur in stark schwefelsaurer Lösung erhalten werden konnten. Die Lösung des Kupfersalzes ist tiefblau, die des Fisenoxydulsalzes braunrot gefürbt. Sowohl die freie Säure wie allch ihre Salze werden durch Zusatz von Wasser unter Entbindung von Stickoxyd zerlegt. Durch Sauerstoff, Salpetersïure, Kaliumpermanganat und andere Oxydationsmittel wird sie in Nitrosylschwefelsäure übergeführt. Die blaue Säure kann aus dieser außer $\mathrm{SO}_{2}$ durch verschiedene andere Reduktionsmittel erhalten werden, durch Eisen, Silber, Zinn, Aluminium und besonders durch Quecksilber. Sie entsteht daher auch bei der Bestimmung des Nitrosegehaltes in der nitrosen Säure mittels des $\mathrm{L} \mathrm{u} \mathrm{n}$ g e schen Nitrometers.

Ihre Kupferverbindung läßt sich, wie ich gefunden habe, in sehr einfacher Weise durch Reduktion von salpetriger oder Salpetersäure in stark schwefelsaurer Lösung mittels Alkohol bei Gegenwart von Kupfersulfat in folgender Weise erhalten. In einem Reagensglase werden $10 \mathrm{ccm}$ konz. Schwefelsäure (spez. Gew. 1,84) mit je zwei Tropfen Weingeist und 25\%iger Salpetersäure gemischt, und die Mischung mit $1 \mathrm{ccm}$ einer Kupfersulfatlösung (1 : 10) überschichtet. Wenn man jetzt das Reagensglas vorsichtig schüttelt, so entsteht an der Berührungszone der beiden Flüssigkeiten ein blauer Ring, und bei stärkerem Schütteln färbt sich die Mischung blau. Gleichzeitig entweicht NO in zahlreichen Bläschen. Besser noch gelingt der Versuch mit gesättigter Kupfersulfatlösung. Man mischt dieselbe direkt mit der Schwefelsäure und fügt dann die Salpetersäure und den Weingeist hinzu. Da eine Reduktion der Salpetersäure wegen der großen Konzentration der Schwefelsäure nicht erfolgen kann, so tritt eine Blaufärbung zunächst nicht auf. Erst wenn man einige Tropfen Wasser zufügt und umschüttelt, färbt sich die Mischung jetzt, und zwar tiefblau. Die Stickoxydentwicklung ist in diesem Falle geringer, weil die Beständigkeit der blauen Säure infolge der größeren Konzentration der Kupfersulfatlösung erheblich größer ist. Daß lediglich die hohe Konzentration der Schwefelsäure die Reduktion der Salpetersäure und die Bildung der blauen Säure in diesem Falle verhindert hat, beweist der folgender Versuch.

Man löst etwas Natriumnitrit in $10 \mathrm{ccm}$ konz. Schwefelsäure (spez. Gew. 1,84), fügt $1 \mathrm{ccm}$ gesättigte Kupfersulfatlösung und einige Tropfen Weingeist hinzu. Jetzt tritt die Blaufärbung sofort ohne Zusatz von Wasser ein.

Hinsichtlich der Zusammensetzung der blauen Verbindung gehen die Ansichten auseinander. S a batior und Tra u $\mathrm{T}$ haben sie als Nitrosodisulfosäure $\mathrm{ON}=\left(\mathrm{SO}_{3} \mathrm{H}\right)_{2}$ angesprochen, $\left.\mathrm{Trautz} \mathrm{z}^{5}\right)$ erklärte die Reduktion der Nitrosylschwefelsäure durch Schwefeldioxyd, z. B. im Gloverturm nach folgenden Gleichungen

$$
\begin{aligned}
& 2 \mathrm{NO}_{2} \mathrm{SO}_{3} \mathrm{H}+3 \mathrm{SO}_{2}+2 \mathrm{H}_{2} \mathrm{O} \\
& =2 \mathrm{NO}<{ }_{\mathrm{SO}_{3} \mathrm{H}}^{\mathrm{SO}_{3} \mathrm{H}}+\mathrm{H}_{2} \mathrm{SO}_{4} \\
& \mathrm{NO}<\frac{\mathrm{SO}_{3} \mathrm{H}}{\mathrm{SO}_{3} \mathrm{H}}+2 \mathrm{NO}_{2} \mathrm{SO}_{3} \mathrm{H}+2 \mathrm{H}_{2} \mathrm{O} \\
& =3 \mathrm{NO}+4 \mathrm{H}_{2} \mathrm{SO}_{4} \text {. }
\end{aligned}
$$

5) Z. f. physikal. Chem. 1904, 602. 
$\mathrm{R}$ a s c h i $\mathrm{g}^{6}$ ) der sich eingehend mit der blauen Säure beschäftigt hat, wies darauf hin, daß diese nicht Nitrosodisulfosäure sein kann, weil sie sonst bei der Spaltung nicht $\mathrm{NO}$ und $\mathrm{H}_{2} \mathrm{SO}_{4}$, sondern entweder $\mathrm{NO}, \mathrm{H}_{2} \mathrm{SO}_{4}$ und $\mathrm{SO}_{2}$ oder $\mathrm{N}$ und $\mathrm{H}_{2} \mathrm{SO}_{4}$ liefern müsse. Nach $R$ a s c $h$ ig s Ansicht ist die blaue Verbindung, eine zweibasische Säure von der Forme]

$$
\mathrm{ON}<\begin{aligned}
& \mathrm{OH} \\
& \mathrm{SO}_{3} \mathrm{H}^{\prime}
\end{aligned}
$$

er nennt sie Nitrosisulfosäure. Ihr Kupfersalz soll folgendermaßen zusammengesetzt sein:<smiles>O=[Se]1C2ON21</smiles>

Die Versuche, die $\mathrm{R}$ a s o $\mathrm{h}$ ig zu dieser Auffassung gefuihrt haben, sind aber nicht eindeutig, und ich vermag seine Schlüsse nicht als richtig anzuerkennen.

Das Kupfersalz der blauen Säure stellt $\mathrm{R}$ a s c h ig u. a. durch Reduktion von Nitrosylschwefelsäure, die in konz. kupfersulfathaltiger Schwefelsäure gelöst war, mittels Quecksilber dar. In diesem Falle müßten sich, wenn die Ansicht $R$ a s c h i g 8 zutreffend wäre, folgende Reaktionen abspielen:

$$
\mathrm{NO}_{2} \mathrm{SO}_{3} \mathrm{H}+\mathrm{H}=\mathrm{ON} / \mathrm{SO}_{3} \mathrm{H}
$$

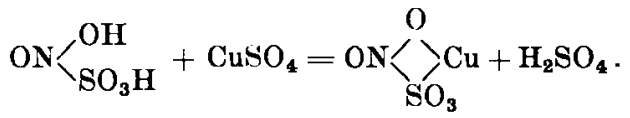

Man kann die Kupferverbindung der blauen Säure auch noch auf anderem Wege erhalten, nämlich durch Auflösen von Stickstoffoxyd in kupfersulfathaltiger Schwefelsäure, was sich mittels des Nitrometers von $\mathrm{Lu}$ g e bequem demonstrieren läßt. Zu dem Zwecke bringt man eine Lösung von $0,05 \mathrm{~g}$ Natriumnitrit in $5 \mathrm{ccm}$ konz. Schwefelsäure in das Nitrometer und reduziert die salpetrige Säure in bekannter Weise durch Schütteln mit Quecksilber. LäBt man nach beendigter Reduktion etwas konz. Kupfersulfatlösung in das Nitrometer fließen und schüttelt gut um, so löst sich das gebildete NO zum Teil in der Schwefelsäure auf, und färbt diese sich dunkelblau. Fügt man noch einige Kubikzentimeter Schwefelsäure hinzu und schüttelt wiederum, so geht weiter Stickoxyd in Lösung.

Die Beständigkeit des Kupfersalzes der blauen Verbindung ist somit abhängig von der Menge der Schwefelsäure, sie ist ferner abhängig von der Konzentration der Schwefelsäure und, wie wir uben bei der Reduktion der Salpetersäure durch Alkohol gesehen haben, auch von der Konzentration der Kupfersulfatlösung. Sofern dem Kupfersalze der blauen Säure die Formel<smiles></smiles>

zukommt, wie $\mathrm{R}$ a s chig glaubt, so müssen bei dem oben beschriebenen Versuche im Nitrometer folgende Reaktionen stattfinden:

6) Diese Z. 18, 1306 (1905).

$$
\mathrm{NO}+\mathrm{H}_{2} \mathrm{SO}_{4}=\mathrm{ON}<\begin{aligned}
& \mathrm{OH} \\
& \mathrm{SO}_{3} \mathrm{H}
\end{aligned}
$$

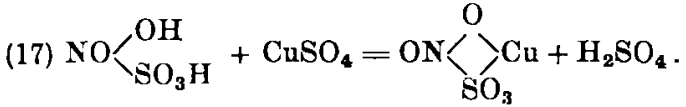

In diesem Falle wäre, wie bei der Reaktion (15) durch die notorisch äußerst labile blaue Säure aus Kupfersulfatlösung Schwefelsäure in Freiheit gesetzt worden. Diese Reaktion erscheint mir höchst unwahrscheinlich, weil sie mit der Affinitätslehre in direktem Widerspruche steht.

Wie ist nun aber die Bildung des Kupfersalzes der blauen Säure bei der Reduktion der Nitrosylschwefelsäure bei Gegenwart von Kupfersulfat oder bei der Auflösung von Stickstoffoxyd in kupfersulfathaltiger Schwefelsäure zu erklüren? Man könnte zunächst annehmen, es handele sich in jedem Falle nicht um eine chemische Reaktion, sondern es liege nur-eine einfache Auflösung von Stickstoffoxyd in schwefelsaurer Kupfersulfatlösung vor. Eine solche Annahme wäre berechtigt, um so mehr, als man die rotbraune Flüssigkeit, welche man beim Einleiten von Stickstoffoxyd in Ferrosulfatlösung erhält, ebenfalls bis vor kurzem als eine Lösung von Stickoxyd aufgefaßt hat. Nach meiner Ansicht handelt es sich hier jedoch nicht um eine einfache Lösung, sondern es liegt tatsächlich das Salz einer Säure vor, die sowohl bei der Reduktion von Nitrosylsohwefelsüure wie bei der Auflösung von Stickoxyd in kupfersulfathaltiger Schwefelsäure entsteht. In dieser Ansicht werde ich dadurch bestärkt, daß sich aus der Lösung des Kupfersalzes pach einiger Zeit violettrote Krystalle abscheiden. Möglicherweisc liertt hier ein Doppelsalz vor. Die Untersuchungen darüber sind jedoch noch nicht abgeschlossen, weshalb diese Mitteilung als vorläufig anzusehen ist.

$\mathrm{Da}$ die blaue Verbindung einerseits bei der Oxydation in Nitrosylschwefelsäure übergeht, und dieselbe andererseits unter Abspaltung von Stickstoffoxyd in Schwefelsäure zerfällt, so muß sie zu der Nitrosylschwefelsäure und der Schwefelsäure in naher Beziehung stehen.

Die Konstitution der Nitrosylschwefelsäure ist bekanntlich strittig. $R$ a s c h i g faBt sio als eine Schwefelstickstr,ifsäure auf und nennt sie daher auch Nitrosulfosäure

$$
\mathrm{O}^{\mathrm{NSO}} \mathrm{N}_{3} \mathrm{H} \text {. }
$$

Er schließt auf diese Konstitution besonders deshalb, weil er die Nitrosylschwefelsäure durch Oxydation einer Schwefelstickstoffverbindung der Hydroxylaminmonosulfosäure

$$
\mathrm{OH}_{\mathrm{H}} / \mathrm{NSO}_{3} \mathrm{Na}
$$

mit C a r o scher Säure erhalten hat. Interessant ist, $\mathrm{da} B \mathrm{R}$ a s c h i g hierbei eine vorübergehende Violettfärbung der Reaktionsflüssigkeit erhielt, die jedenfalls auf eine intermediäre Bildung der blauen Säure zurückzuführen ist.

L u n g e erkannte die Schlüsse, die $R$ a s c h i g aus diesem Versuch gezogen hat, nicht als zutreffend an und wies darauf hin, daß nach Analogio anderer von ihm selbst angeführter Reaktionen bei 
der Oxydation von Hydroxylaminmonosulfosäure nicht notwendigerweise eine Nitroverbindung entstehen müsse, ebensogut ist auch die Bildung einer Nitrosoverbindung möglich.

Mit Rücksicht auf die Bildung der Nitrosylschwefelsëure aus Schwefelsānre und Stickstofftrioxyd:

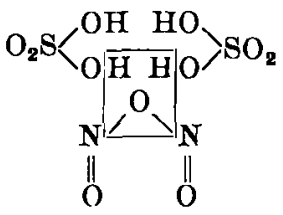

halte ich die Auffassung L u n g e s für die richtige. Der blauen Säure aber kommt meiner Ansicht nach folgende Konstitutionsformel $\mathrm{zu}$ :<smiles></smiles>

Der Körper entsteht bei der Reduktion der Nitrosylschwefelsäure durch Austritt von einem Atom Sauerstoff aus 2 Molekülen, z. B. nach der Gleichung

$$
\begin{aligned}
& \mathrm{O}_{2} \mathrm{~S}\left\langle\begin{array}{l}
\mathrm{OHHO} \\
\mathrm{ONNO}
\end{array} \mathrm{SO}_{2}+\mathrm{H}_{2}\right. \\
& \text { OI } \\
& =\mathrm{O}_{2} \mathrm{~S}\left\langle\begin{array}{l}
\mathrm{OH} \\
\mathrm{ON} \\
\mathrm{O}
\end{array} \mathrm{NO}^{\mathrm{HO}}\right.
\end{aligned}
$$

Die Verbindung ist sehr unbeständig und zerfällt durch Hydrolyse in $\mathrm{H}_{2} \mathrm{SO}_{4}$ und $\mathrm{NO}$ nach

$$
\begin{gathered}
\mathrm{O}_{2} \mathrm{~S}\left\langle\begin{array}{l}
\mathrm{OHI} \\
\mathrm{ONO}-\mathrm{NO}
\end{array}>^{\mathrm{O}} \mathrm{SO}_{2}+\mathrm{H}_{2} \mathrm{O}\right. \\
=2 \mathrm{H}_{2} \mathrm{SO}_{4}+2 \mathrm{NO} .
\end{gathered}
$$

Bei der Oxydation entsteht Nitrosylschwefelsäure nach

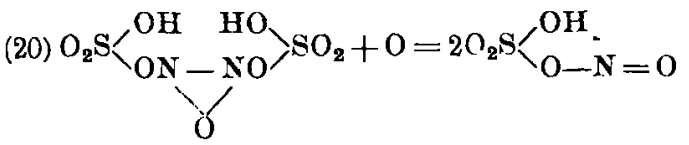

Bei der Annahme dieser Formel läßt sich auch die Bildung der Kupferverbindung aus $\mathrm{NO}, \mathrm{H}_{2} \mathrm{SO}_{4}$ und $\mathrm{CuSO}_{4}$ ungezwungen erklären. Fs ist bekannt, daß das Kupfer leicht komplexe Verbindungen eingeht, deren Lösungen tiefblau gefärbt sind, z. B. alkalische Kupfertartratlösung. Treffen Schwefelsäure, Kupfersulfat und Stickstoffoxyd zusammen, so entsteht zunächst saures Kupfersulfat und hieraus nach

$$
\begin{aligned}
& \mathrm{O}_{2} \mathrm{~S}\left\langle\underset{\mathrm{OH}}{\mathrm{O}-\mathrm{Cu}-\mathrm{O}} \backslash \mathrm{SO}_{2}: 2 \mathrm{NO}\right.
\end{aligned}
$$

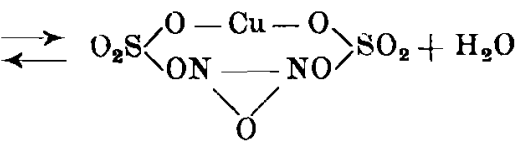

das Kupfersalz der blauen Säure.
Ich werde die blaue Säure wegen ihrer Beziehung zur Nitrosylschwefelsäure für die Folge nitrosylige Schwefelsëure nennen. Die hier gegebene Konstitutionsformel ist zwar ebenso hypothetisch, wie die der Nitrosisulfosäure $R$ a s c h i g s und der Sulfonitronsäure $\mathrm{L}$ u n g es, sie erscheint mir jedoch wahrscheinlicher wie die von beiden Autoren angenommene Formel

$$
\mathrm{O}=\mathrm{N} / \begin{aligned}
& \mathrm{OH} \\
& \mathrm{SO}_{\mathbf{3}} \mathrm{H}
\end{aligned}
$$

Wie die Nitrosisulfosäure (Sulfonitronsäure) ist die nitrosylige Schwefelsäure ebenfalls eine zweibasische Säure, und. sie erfordert zur Utberführung in Nitrosylschwefelsäure genau soviel Sauerstoff wie die Nitrosisulfosäure (Sulfonitronsäure), so daß auch die analytischen Befundo $R$ ascli igs mit der von mir aufgestellten Formel übereinstimmen.

Zur Erklärung des Bleikammerprozesses ist die blaue Säure zuerst im Jahre 1904 von $T$ r a $u t^{7}$ ) herangezogen worden, der sie allerdings, wie schon gesagt, als Stickoxyddisulfosäure auffaßte. Außer den unter (12) und (13) angegebenen Reaktionen häit T r a u t z noch folgende für möglich:

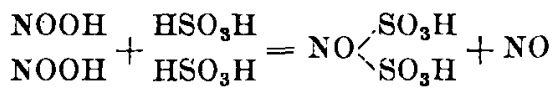

$\mathbf{R}$ a s c h i g ist dann in dessen Fußstapfen getreten. Bereits im Jahre 1887 hat $\mathrm{R}$ a $8 \mathrm{c} \mathrm{h} \mathrm{i} \mathrm{g}^{8}$ ) eine Theorie des Bleikammerprozesses veröffentlicht, in der als Zwischenstufe die hypothetische Dihydroxylaminsulfosäure $\left.(\mathrm{OH})_{2} \mathrm{NSO}_{3} \mathrm{H}\right)$ auftritt. Folgende Gleichungen geben die damaligen Anschauungen $R$ a s c h i g s wieder.

(23) $\mathrm{N}(\mathrm{OH})_{3}+\mathrm{HSO}_{3} \mathrm{H}=(\mathrm{OH})_{2} \mathrm{NSO}_{3} \mathrm{H}+\mathrm{H}_{2} \mathrm{O}$,

(24) $(\mathrm{OH})_{2} \mathrm{NSO}_{3} \mathrm{H}+\mathrm{N}(\mathrm{OH})_{3}$

$$
=2 \mathrm{NO}+\mathrm{H}_{2} \mathrm{SO}_{4}+2 \mathrm{H}_{2} \mathrm{O}
$$

(25) $2 \mathrm{NO}+\mathrm{O}+3 \mathrm{H}_{2} \mathrm{O}=2 \mathrm{~N}(\mathrm{OH})_{3}$.

Fortgesetzte Studien über den Bleikammerprozeß 3 und besonders die blaue Säure führten R a s c h i g ${ }^{9}$ ) dazu, diesen Körper als weitere Zwischenstufe aufzunehmen. In der neuen Theorie $R$ a s chigs erscheint die Dihydroxylaminsulfosäure als Nitrososulfosäure $\left(\mathrm{HSNO}_{4}\right)$. Die Theorie selbst ist durch die nachstehenden Gleichungen ausgedrückt.

(26) $\mathrm{HNO}_{2}+\mathrm{SO}_{2}=\mathrm{HSNO}_{4}$,

(27) $\mathrm{HSNO}_{4}+\mathrm{HNO}_{2}=\mathrm{H}_{2} \mathrm{SNO}_{5}+\mathrm{NO}$,

(28) $\mathrm{H}_{2} \mathrm{SNO}_{5}=\mathrm{H}_{2} \mathrm{SO}_{4}+\mathrm{NO}$,

(29) $2 \mathrm{NO}+\mathrm{O}+\mathrm{H}_{2} \mathrm{O}=2 \mathrm{HNO}_{2}$.

Die Arbeiten $\mathrm{R}$ as chigs über die blaue Säure haben Lunge und Be r ${ }^{10}$ ) im Jahre 1906 veranlaßt, ebenfalls diese Verbindung als Zwischenstufe zu akzeptieren. Für $\mathrm{L}$ u n $\mathrm{g}$ e bedeutet dies die Aufgabe seines bis dahin energisch vertretenen Standpunktes: es könne keine Theorie als richtig betrachtet werden, welche auf die Reduktion der Salpetergase bis auf Stickoxyd gegründet ist.

Die nachstehenden Gleichungen geben die jetzigen Anschauungen $L$ ung es wieder.

7) 1. c.

8) Liebigs Ann. 241, 242.

9) Diese Z. 18, 1310 (1905).

10) Diese Z. 19, 887 (1906). 
(30) $\mathrm{SO}_{2}+\mathrm{NO}_{2}+\mathrm{H}_{2} \mathrm{O}=\mathrm{SO}_{5} \mathrm{NH}_{2}$,

(31a) $2 \mathrm{SO}_{5} \mathrm{NH}_{2}+\mathrm{O}=\mathrm{H}_{2} \mathrm{O}+2 \mathrm{SO}_{5,2} \mathrm{NH}$,

(3Ib) $2 \mathrm{SO}_{5} \mathrm{NH}_{2}+\mathrm{NO}_{2}=2 \mathrm{SO}_{5} \mathrm{NH}+\mathrm{NO}+\mathrm{H}_{2} \mathrm{O}$,

(32a) $2 \mathrm{SO}_{2} \mathrm{NH}+\mathrm{H}_{2} \mathrm{O}=2 \mathrm{H}_{2} \mathrm{SO}_{4}+\mathrm{NO}+\mathrm{NO}_{2}$,

(32b) $2 \mathrm{SO}_{\overline{5}} \mathrm{NH}+\mathrm{SO}_{2}+2 \mathrm{H}_{2} \mathrm{O}=\mathrm{H}_{2} \mathrm{SO}_{4}+2 \mathrm{SO}_{i} \mathrm{NH}_{2}$

(33) $\mathrm{SO}_{5} \mathrm{NH}_{2}=\mathrm{NO}+\mathrm{H}_{2} \mathrm{SO}_{4}$,

(34) $2 \mathrm{NO}+\mathrm{O}_{2}=\mathrm{N}_{2} \mathrm{O}_{4}$.

Trotzdem die Davy sche Theorie viele Anhänger gefúnden, hat es niemals an Gegnern gefehlt. B e r z e li u s erklärte in seinem Lehrbuche die Bildung der Bleikammerkrystalle für einen Ausnahmefall, der bei Wassermangel in der Kammer eintrete.

Er selbst erklärte den Bleikammerprozeß entsprechend folgenden Gleichungen:

(35) $\mathrm{SO}_{2}+\mathrm{N}_{2} \mathrm{O}_{3}+\mathrm{H}_{2} \mathrm{O}=\mathrm{H}_{2} \mathrm{SO}_{4}+2 \mathrm{NO}$,

(36) $2 \mathrm{NO}+\mathrm{O}=\mathrm{N}_{2} \mathrm{O}_{3}$.

Welche Gründe B e r z e l i u s veranlaßt haben, die Nitrosylschwefelsäure als Zwischenstufe abzulehnen, geht aus der Literatur nicht hervor. Tra u tz glaubt, daß ihn ähnliche Ưberlegungen dazu geführt haben, wie die gewesen sind, die $\mathrm{O}$ s t w a ld später in Beziehung auf $Z$ wischenreaktionen durchgeführt habe, d. h., daß Nebenprodukte keine Zwischenprodukte zu sein brauchen.

Positiver drückt sich J uris ch ${ }^{11}$ ) aus, er sagt zu der L u n g e schen Theorie: „Sie gründet sich auf die Zwangsvorstellung, daß der Körper $\mathrm{HSNO}_{5}$ unter denselben Umständen entstehe, unter welchen er sofort zerlegt wird. Selbst wenn man den Körper $\mathrm{HSNO}_{5}$ als eine katalytische $\mathrm{Z}_{\text {wischenstufe betrach- }}$ ten will, so ist seine Annabme überflüssig, weil in den einfachen nitrosen Verbindungen $\mathrm{N}_{\mathbf{x}} \mathrm{O}_{\mathbf{y}}$ gènügend wirksame katalytische Substanzen vorhanden sind."

Allerdings, wenn tatsächlich die direkte Bildung der Schwefelsäure nach den Gleichungen:

(37) $\mathrm{SO}_{2}+\mathrm{NO}_{2}+\mathrm{H}_{2} \mathrm{O}=\mathrm{H}_{2} \mathrm{SO}_{4}+\mathrm{NO}$ oder

(38) $\mathrm{SO}_{2}+\mathrm{N}_{2} \mathrm{O}_{3}+\mathrm{H}_{2} \mathrm{O}=\mathrm{H}_{2} \mathrm{SO}_{4}+2 \mathrm{NO}$

erfolgen kann, dann erscheint die indirekte Bildung über eine oder gar zwei Zwischenstufen sehr unwahrscheinlich. Es ist nicht anzunehmen, $\mathrm{da} B$ in diesem Falle die Natur den Umweg über die Nitrosylsch wefelsäure einschlagen und diesen dem direkten Weg vorziehen wird.

Die Nitrosylschwefelsäure wird wohl nur darum als Zwischenstufe angesehen, weil sie sich fast momentan bildet, wenn $\mathrm{SO}_{2}, \mathrm{O}$, NO und wenig Wasser zusammentreffen. $J$ u ri s c h (loc. cit.) führt zehn verschiedene Reaktionen auf, nach denen in der Bleikammer Nitrosylschwefelsäure primär oder sekundär entstehen kann. Nach meiner Ansicht sind alle diese Reaktionen auf zwei zurückzuführen, die nach folgenden Gleichungen verlaufen:

(39) $\mathrm{SO}_{2}+\mathrm{NO}_{2}+\mathrm{H}_{2} \mathrm{O}=\mathrm{H}_{2} \mathrm{SO}_{4}+\mathrm{NO}$,

(40) $2 \mathrm{H}_{2} \mathrm{SO}_{4}+\mathrm{NO}+\mathrm{NO}_{2}=2 \mathrm{HSNO}_{5}+\mathrm{H}_{2} \mathrm{O}$.

DaB in der Tat die Bildung der Schwefelsäure beim Bleikammerproze $B$ in der Hauptsache entsprechend der Theorie von B e rzeli u s, d. h., daß die Oxydation des $\mathrm{SO}_{2}$ in der Kammer durch Stickstoffoxyde unmittelbar erfolgt, und daß ferner weder

11) Chem. Industr. 1910. die Nitrosylschwefelsäure, noch die nitrosylige Schwefelsäure (Sulfonitronsäure, Nitrosisulfosäure) als Zwischenstufe bei dem Prozesse auftreten, dies scheint mir aus den nachstehend mitgeteilten Versuchen hervorzugehen.

Bei diesen Versuchen benutzte ich den auf S.1708 abgebildeten Demonstrationsapparat zur Darstellung von Schwefelsäure. Derselbe beruht auf dem Prinzip des bekannten Turmverf. und besteht in der Hauptsache aus drei Glaszylindern, wie ich einen solchen bereits beschrieben habe. Die Zylinder sind durch Verbindungsstücke so geschaltet, dab die eingeführten Reaktionsgase nacheinander den ersten, dann den zweiten und drittein $Z y$ linder passieren. Diese sind zweckentsprechend auf einer mit Rädern versehenen Unterlage angeordnet, die, wenn Kühlung beabsichtigt wird, aus einem Blechkasten, sonst aus einem Brett besteht. Die Räder sind so befestigt, daß sich durch kurzes Hinund Herschieben des Apparates Schüttelbewegungen ausführen lassen. Selbstredend kann der Apparat auch auf einer besonderen Schüttelvorrichtung montiert werden. Die Darstellung der Schwefelsäure erfolgt im ersten Zylinder, wo ein Gemisch von Schwefeldioxyd und Luft auf nitrose Säure einwirkt. Die übrigen Zylinder dienen zur Absorption der dabei entstehenden nitrosen Gase und werden zu dem $Z$ wecke mit konz. Schwefelsäure beschickt. Die für die vorliegenden Versuche benutzte Nitrose hatte das spez. Gew. 1,5. Bekanntlich ist die Beständigkeit der in Schwefelsäure gelösten Nitrosylschwefelsäure abhängig von dem Wassergehalte der Schwefelsäure oder, mit anderen Worten, es ist die Spannkraft der salpetrigen Säure in schwefelsaurer Lösung um so größer, je höher der Wassergehalt der Schwefelsäure ist. Eine Nitrose vom spez Gow. 1,5 enthält viel freie salpetrige Sïure, sie ist gelb gefärbt und entwickelt beim Schütteln reichliche Mengen nitroser Gase. Daher war auch der Gasraum des Denitrierzylinders, nachdem er zu drei Vierteln mit Nitrose beschickt worden, mit nitrosen Gasen angefüllt und dadurch gelbbraun gefärbt. Beim Einleiten des Schwefeldioxyd-Luftgemisches trat im vorderen Teile des Cylinders sofort eine Entfärbung des Gasraumes ein. Die Entfärbung schritt schnell nach hinten fort und in wenigen Augenblicken war der Gasraum farblos geworden. Wurde der Apparat jetzt geschüttelt, so färbte sich der Gasraum sofort wieder gelbbraun, ausgenommen der vordere Teil, wo $\mathrm{SO}_{2}$ in UtberschuB vorhanden war. Bei fortgesetztem Schütteln blieb auch der Gasraum gefärbt, solange noch erhebliche Mengen von freier salpetriger Säure in der Nitrose enthalten waren. Letztere wurde mit fortschreitender Denitrierung mehr und mehr entfärbt. Das Charakteristische des Vorganges ist, $\mathrm{da} B$ bei der Denitrierung der Nitrose eine Blaufärbung derselben durch Bildung von nitrosyliger Schwefelsäure und Entwicklung von Stickstoftoxydbläschen i $n \mathbf{n}$ e $\mathbf{h}$ a l b der Säure erst beobachtet werden konnte, nachdem die Denitrierung fast beendigt war. Die Blaufärbung der Säure und Stickstoffoxydentwicklung war zudem ganz gering.

Bei der Denitrierung einer Nitrose vom spez. Gew. 1,5 spielen sich in dem Zylinder die Vorgänge des Gloverturmes und der Bleikammer ab. Allerdings erfolgt die Schwefelsäurebildung nicht ganz wank Blofitatha und Gloverturmbedingungen, 
denn das Schwefeldioxyd kommt in kaltem Zustande mit der nitrosen Säure und den nitrosen Gasen in Berührung, auch wird kein Wasserdampf eingeführt.

Wenn die Denitrierung der nitrosen Säure trotz der niedrigen Temperatur schnell verläuft, so liegt dies, was ich wohl nicht mehr zu sagen brauchte, an dem hohen Wassergehalte der Nitrose und der großen Spannkraft der salpetrigen Säure, Hieraus ist es jedenfalls auch zu erklären, daß bei der Denitrierung eine Bildung von nitrolysiger Schwefelsäure resp. Entwicklung von Stickstoffoxyd innerhalb der Flüssigkeit erst kurz vor Beendigung der Denitrierung und auch dann nur in unerheblichem $\mathrm{Ma}$ (e eintritt. Bei der Einwirkung von $\mathrm{SO}_{2}$ auf Nitrose vom spez. Gew. 1,71 tritt die Blaufärbung und Stickoxydentwicklung auch bei Gegenwart von Luft sofort ein.

Der Unterschied zwischen den beiden beschriebenen Versuchen besteht darin, daß im ersten Falle $\mathrm{SO}_{2}$ auf Nitrosylschwefelsäure und beim zweiten Versuche auf salpetrige Säure, sowie nitrose Gase, die ja beim Schütteln des Apparates frei werden, einwirkte. Auch in der schwächeren Säure bildet sich, wie gesagt, nitrosylige Schwefelsäure, aber erst dann, wenn die Nitrose nahezu denitriert ist, d. h., wenn keine freie salpetrige Säure mehr vorhanden ist, sondern nur noch Nitrosylschwefelsäure.

Die Annahme $R$ as chigs und LungeBerls, beim Zusammentreffen von $\mathrm{SO}_{2}$ mit salpetriger Säure bzw. $\mathrm{NO}_{2}$ und Wasser entstehe Sulfonitronsäure (Nitrosisulfosäure), scheint mir durch den letzten Versuch schon widerlegt. Die freie nitrosylige Sehwefelsäure bildet sich nur bei der Reduktion der Nitrosylschwefelsäure, und zwar im vorliegenden Falle nach der Gleichung:

$$
\begin{aligned}
& 2 \mathrm{HSNO}_{5}+\mathrm{SO}_{2}+\mathrm{H}_{2} \mathrm{O}
\end{aligned}
$$

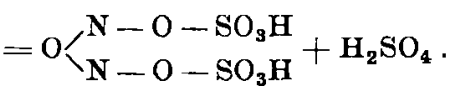

$\mathbf{L} \mathbf{u}$ g e sagt in seinem Handbuch der Schwefelsäurefabrikation (1903, S. 185): „In vollkommen trockenem Zustande wirkt Schwefeldioxyd nicht auf die trockenen Stickoxyde, aber in Gegenwart der geringsten Mengen von Wasser entstehen Kammerkrystalle, wenn $\mathrm{SO}_{2}$ mit $\mathrm{N}_{2} \mathrm{O}_{4}$ oder aber mit $\mathrm{NO}$ oder $\mathrm{N}_{2} \mathrm{O}_{3}$ und zugleich mit Sauerstoff zusammentrifft." Es entspricht dies den Beobachtungen, wie sie z. B. bei dem bekannten Vorlesungsversuche gemacht worden, durch den im Anschlusse an die $\mathrm{Er}$ klärung des Bleikammerprozesses die Bildung der Bleikammerkrystalle gezeigt $\mathrm{zu}$ werden pilegt. Obige Behauptung $\mathrm{L}$ u $\mathrm{ng} \mathrm{e}$ ist zweifellos richtig, nur müssen die Worte, ,geringste Mengen von Wasser" richtig aufgefaßt werden. Falsch wäre es natürlich, den Satz dahin zu interpretieren, als ob schon bei Anwesenheit minimaler Mengen von Wasser erhebliche Quantitäten Nitrosylschwefelsäure gebildet werden könnten. Die Menge der gebildeten Nitrosylsäure steht zu der vorhandenen Wassermenge selbstredend in molekularem Verhältnis.

In meinem Apparate erfolgte die Reaktion ohne Zuführung von Wasser. Da das Schwefeldioxyd-Luftgemisch vor dem Eintritt in den Apparat durch Schwefelsäure getrocknet wurde, und die Dampfspannung einer 60\%igen Schwefelsäure, wie sie hier vorliegt, nach $S$ or e 11 bei $20^{\circ}$ nur $3 \mathrm{~mm}$ heträgt, so kann der Wassergehalt im Gasraum des Apparates nur sehr gering sein. Selbst wenn bei der Denitrierung der Nitrose die Temperatur im Apparate auf $40^{\circ}$ steigt, beträgt die Dampfspannung der Schwefelsäure nur $10 \mathrm{~mm}$, was einem Wassergehalte von ca. $1 \mathrm{mg}$ in $100 \mathrm{ccm}$ der Reaktionsgase entspricht. Hieraus ergibt sich, daß in dem Apparate nennenswerte Mengen von Nitrosylschwefelsä.ure durch Kondensation aus $\mathrm{SO}_{2}, \mathrm{NO}_{2}$ oder $\mathrm{N}_{2} \mathrm{O}_{3}$, und $\mathrm{H}_{2} \mathrm{O}$, sofern überhàupt eine Kondensation, analog der Davyschen Theorie stattfindet, nicht gebildet werden können. Dasselbe gilt auch für jeden anderen angenommenen $Z$ wischenkörper, welcher $\mathrm{HNO}_{2}, \mathrm{H}_{2} \mathrm{SO}_{3}$ als molekulare Koeffizienten voraussetzt. $\mathrm{Da}$ ich aber nun leicht nachweisen kann, daß durch die Reaktion im Gasraum meines Apparates relativ große Mengen Schwefelsäure gebildet werden, so kann dies nur durch direkte Oxydation des Schwefeldioxyds durch die nitrosen Gase geschehen, entsprechend der Gleichung:

(42) $\mathrm{SO}_{2}+\mathrm{NO}_{2}=\mathrm{SO}_{3}+\mathrm{NO}$

Das gebildete Schwefeltrioxyd löst sich weiter in der Bodensäure beim Schïtteln des Apparates auf, und das Stickstoffoxyd wird durch den Luftsauerstoff in $\mathrm{NO}_{2}$ übergeführt.

Nach meiner Ansicht entsteht auch bei Anwesenheit größerer Mengen von Wasser z. B. in der Bleikammer bei der Einwirkung von $\mathrm{SO}_{2}$ auf $\mathrm{NO}_{2}$ primär stets $\mathrm{SO}_{3}$ bzw. $\mathrm{H}_{2} \mathrm{SO}_{4}$ und nicht Nitrosylschwefelsäure. Wenn letztere in der Kammer, z.B. bei Wassermangel auftritt, so ist sie das Produkt eines sekundären Vorganges, d. h. durch Einwirkung von nitrosen Gasen auf Schwefelsäure entstanden nach der Gleichung:

(43) $2 \mathrm{H}_{2} \mathrm{SO}_{4}+\mathrm{NO}+\mathrm{NO}_{2}=2 \mathrm{HSNO}_{5}+\mathrm{H}_{2} \mathrm{O}$.

Dieser Vorgang läßt sich gleichfalls mittels meines Schwefelsäureapparates veranschaulichen. Der Zylinder 1 wird wiederum mit Nitrose vom spez. Gew. 1,5 beschickt, und eines der beiden Kugelrohre mittels eines Stückchens Gummischlauches und Quetschhahn verschlossen. Auf den Tubus des Zylinders wird das U-Rohr c (s. Abb.) aufgesetzt. Das U-Rohr steht mit einer Waschflasche d, in der sich konz. Schwefelsäure befindet, in Verbindung، Die Wandungen des einen Schenkels des gut ausgetrockneten U-Rohres wird mit 60grädiger Schwefelsäure befeuchtet. Wenn man jetzt durch Schütteln des Apparates nitrose Gase entwickelt und diese durch einen mittels Schwefelsäure getrockneten Luftstrom durch das U-Rohr führt, so lösen sich die nitrosen Gase in der Schwefelsäure auf, und es entsteht nitrose Säure. Läßt man jetzt durch den Zylinder und das U-Rohr Schwefeldioxyd strömen, so wird die in der Nitrose enthaltene Nitrosylschwefelsäure zum Teil reduziert. Man erkennt dies an dem Auftreten zahlreicher Bläschen von Stickoxyd. Das bei der Reduktion entstandene Schwefeltrioxyd löst sich in der Schwefelsäure auf, wodurch deren Konzentration erhöht wird. Weńn man das Experiment mehrmals wiederholt, so wird die Konzentration der Säure schließlich so groß, daß beim Überleiten der nitrosen Gase Nitrósylschwefelsäure auskrystallisiert, und es erscheint dann der eine Schenkel mit Kammerkrystallen bedeckt. In dem nicht mit Schwefelsäure befeuchteten Schenkel entstehen keine Kammer- 
krystalle; jedoch überziehen sich dessen Wandungen allmählich mit einer dünnen, weißen, körnigen Schicht. Es ist dies ebenfalls Nitrosylschwefelsäure, die sich sekundär unter Mitwirkung des geringen Wassergehaltes der Reaktionsgase bildet. Es handelt sich, wie ich durch Analyse des Belags wiederholt festgestellt habe, aber dabei nicht um reine Nitrosylschwefelsäure, sondern um ein Gemenge von Nitrosylschwefelsäure mit Schwefelsäure.

Um die Zusammensetzung des Belags zu mitteln, wurde statt des U-Rohres ein gut ausgetrockneter, an beiden Enden mit Hähnen versehener Glaszylinder auf den Tubus aufgesetzt unter Zwischenschaltung eines trockenen Kugelrobres, In den Zylinder, welcher ebenfalls, um feuchte Luft fernzuhalten, an eine mit konz. Schwefelsäure beschickte Waschflasche angeschlossen war, wurden abwechselnd Schwefeldioxyd und nitrose Gase eingeleitet, bis sich an den Wandungen genügend Nitrosylschwefelsäure gebildet hatte. Dann wurden die Hähno des Zylinders geschlossen, und nachdem dieser von dem Apparate getrennt worden, zur Entfernung der nitrosen Gase resp. des $\mathbf{S O}_{2}$ kurze Zeit trockene Luft hindurch geleitet. Hierauf brachte ich mit Hilfe eines Trichterchens $20 \mathrm{ccm}$ n. Natronlauge in den Zylinder und spülte damit den Belag von den Wandungen ab. Die erhaltene Lösung wurde in einen Kolben übergeführt, mit Wasser auf $100 \mathrm{ccm}$ gebracht und dann halbiert. In der einen Hälfte bestimmte ich die Schwefelsëure als Bariumsulfat, in der anderen die salpetrige Säure mittels Kaliumpermanganats. Die Resultate wurden auf $\mathrm{SO}_{3}(\mathbf{x})$ oder Nitrosylschwefelsäure umgerechnet. Aus der Menge der letzteren berechnete ich weiter, wieviel $\mathrm{SO}_{3}(y)$ zur Bildung der Nitrosylschwefelsäure erforderlich. Aus dem Verhältnis $\mathbf{x}: \mathbf{y}$ ergab sich dann, ob der Belag nur aus Nitrosylschwefelsäure bestand oder ob ein Gemenge von Nitrosylschwefelsäure und Schwefelsäure vorlag. Stets wurde $\mathbf{x}$ größer als y gefunden, meist betrug $x$ das Mehrfache von $y$.

tber die Zusammensetzung der Bleikammerkrystalle und der Nitrosylschwefelsäure hat lange Unklarheit bestanden. Es ist dies durchaus erklärlich. Die Bleikammerkrystalle sind, sofern sie aus $\mathrm{SO}_{2}$, NO, $\mathrm{O}$ und $\mathrm{H}_{2} \mathrm{O}$ erzeugt werden, niemals konstant zusammengesetzt, weil sie eben sekundär in der eben beschriebenen Weise entstehen, und der Gehalt an Nitrosylschwefelsäure von.der Daver der Einwirkung der $\mathrm{SO}_{2}$ abhängt. Übrigens ist es möglich, auch die Bleikammerkrystalle durch tberleiten von Schwefeldioxyd vollständig $\mathrm{zu}$ denitrieren. Bei der Denitrierung tritt eine Blaufärbung der Krystalle ein, es entsteht also dabei gleichfalls nitrosylige Schwefelsäure.

Um das Gelingen der Bestimmung der Zusam. mensetzung des Belages zu sichern, ist es zweckmäßig, mit möglichst trockenen Gasen zu arbeiten. Daher verwendete ich bei dieser Bestimmung zur Erzeugung der nitrosen Gase nicht 48grädige nitrose Säure, sondern ein Gemenge von Schwefelsäure und Salpetersäure, hergestellt aus 800 g 60 grädiger Schwefelsäure und $50 \mathrm{ccm}$ 60\%iger Salpetersäure. Hieraus erhält man bei Behandlung mit Schwefeldioxyd und Luft genügend trockene nitrose Gase.

Da die D a y sche Theorie und ebenso alle anderen Theorien, bei denen die Nitrosylschwefelsäure als Zwischenstufe auftritt, sich auf die Bildung von Bleikammerkrystallen beim Bleikammerprozeß stützen, so wird nach meiner Ansicht duroh diese Versuche den betreffenden Theorien die Basis entzogen. Immerhin beweisen die Versuche nur, daB die Kammerkrystalle sekundär entstehen können, eine primäre Bildung wird dadurch nicht ausgeschlossen. L u $\mathrm{n} g$ e behauptet, es sei in der Bleikammer primär gebildete Nitrosylschwefelsäure als $\mathrm{N}$ e b e l vorhanden, deshalb sollte man annehmen, dieselbe sei dort nachzuweisen. Besonders in meinem Apparate müßte sie, wenn die Annahme $\mathrm{L}$ u n g o s richtig ist, $z u$ finden sein, weil hier wegen des herrschenden Wassermangels eine hydrolytische Spaltung etwa vorhandener Nitrosylschwefelsäure nicht eintreten kann. Ich habe auch in dieser Richtung Versuche angestellt, wobei ich von folgenden Erwägungen ausging. Sofern beim Zusammentreffen von $\mathrm{NO}, \mathrm{O}, \mathrm{H}_{2} \mathrm{O}$ und überschüssigem Schwefeldioxyd (d. h. überschüssig in bezug auf NO) durch KondensationNitrosylschwefelsäure entsteht, so maB erstens beim Einleiten des Reaktionsproduktes in Chlorbariumlösung Bariumsulfat ausfallen und zweitens beim Einleiten der Gase in konz. Schwefelsäure letztere durch Bildung von nitrosyliger Schwofelsäure blaugefärbt werden. Wie ich nicht anders erwartet hatte, fiel bei der Ausführung entsprechender Versuche, wobei Nitrose vom spez. Gew. 1,7 verwendet wurde, nur der erste positiv, der zweite negativ aus. Der letztere wider Erwarten aber auch dann, als ich bei einem Kontrollversuche der Schwefelsäure etwas Natriumnitrit zugesetzt hatte; nur eine Entwicklung von Stickstoffoxyd war hier zu beobachten. Fügte ich jedoch zu der nitrosen Säure etwas konz. Kupfersulfatlösung, so färbte sich die Säure sofort dunkelblau. Beim Durchleiten von $\mathrm{SO}_{2}$ verschwand allmählich aber die Blaufärbung wieder, woraus geschlossen werden $\mathrm{muB}$, daB die nitrosylige Schwefelsäure durch Schwefeldioxyd reduziert wird. Es läßt sich dies auch durch einen einfachen Reagensglasversuch zeigen. Wie oben, stellt man sich zunächst aus $10 \mathrm{ccm}$ konz. Schwelefsäure, konz. Kupfersulfatlösung, Natriumnitrit und Weingeist eine Lösung von nitrosyligsohwefelsaurem Kupfer dar und fügt dann etwas Natriumsulfit in Krystallen hinzu. Schon nach wenigen Augenblikken ist die nitrosylige Schwefelsäure reduziert; dabei die Farbe der Lösung hellblau. Es wird also nicht allein die Nitrosylschwefelsäure, sondern auch die nitrosylige Schwefelsäure durch $\mathrm{SO}_{2}$ reduziert; dabei hindert die Anwesenheit von Sauerstoff die Reduktion nicht, falls überschüssiges Schwefeldioxyd vorhanden ist.

Aus diesen Versuchen ergibt sich zunächst, daß gemäß der Theorie von $L$ u n g e und Ber $l$ Nitrosylschwefelsäure sich in der Bleikammer dort nicht bilden kann, wo $\mathrm{SO}_{2}$ im Uberschuß, d. h. in bezug auf NO im Uberschuß vorhanden ist, weil etwa die in der ersten Phase entatandene Sulfonitronsäure sofort wieder durch $\mathrm{SO}_{\mathbf{z}}$ reduziert werden müßte. Es kann bei UUberschuß von $\mathrm{SO}_{2}$ auch eine Bildung von Schwefelsäure nach der Dav y schen Theorie nicht erfolgen, entsprechend der Gloichung

(44) $2 \mathrm{HSNO}_{5}+\mathrm{H}_{2} \mathrm{O}=2 \mathrm{H}_{2} \mathrm{SO}_{4}+\mathrm{N}_{2} \mathrm{O}_{3}$ 
denn, sofern überhaupt eine Kondensation von $\mathrm{SO}_{2}$, NO, $O$ und Wasser zu Nitrosylschwefelsäure statt. findet, müßte diese. sofort durch Schwefeldioxyd wieder reduziert werden. L u $\mathbf{n}$ g e selbst nimmt ja auch an, daß eine solche Reduktion im vorderen Teile der Kammer erfolgt.

Für vollständig ausgeschlossen halte ich auch einen Reaktionsverlauf nach der Theorie von $R$ a s c h i g bei überschüssigem Schwefeldioxyd. Wenn tatsächlich durch Einwirkung von $\mathrm{SO}_{2}$ auf $\mathrm{HNO}_{2}$ gemäß der Gleichung (26) Nitrosulfosäure gebildet würde, was ich aber aus gewissem Grunde bestreiten muß, so kann die von $R$ a s c hig angenommene Reaktion (27) nicht eintreten, weil bei überschüssigem Schwefeldioxyd kein zweites Molekül $\mathrm{HNO}_{2}$ zur Bildung von Nitrosisulfosäure disponibel ist. Irgend ein Beweis dafür, daß Nitrosisulfosäure (Sulfonitronsäure) in der Bleikammer oder unter Bleikammerbedingungen entsteht, ist weder von $\mathrm{R}$ a s c h i g noch von L u n g e und B e r l erbracht worden.

Ich möchte noch einmal auf die Reduktion der nitrosyligen Schwefelsäure durch Schwefeldioxyd zurückkommen. Dieser Körper wird, wie ich gezeigt habe, sehr leicht beim $\dot{\mathrm{U}}$ b e $r$ l e i t e $\mathrm{n}$ von Schwefeldioxyd über nitrose Süure vom spez. Gew. 1,7 erhalten; daher konnte ich auch beim $\mathrm{E}$ i n1 e i t e $n$ von $\mathrm{SO}_{2}$ in nitrose Säure von gleicher Konzentration eine Blaufürbung derselben durch Bildung von nitrosyliger Schwefelsäure erwarten. Dieser unterschiedliche Reaktionsverlauf bei der Reduktion der Nitrosylschwefelsäure durch Schwefeldioxyd wird durch die Konzentrationsverhältnisse der reagierenden Körper bedingt. Sofern Nitrosylschwefelsäure mit überschüssigem Schwefeldioxyd zusammentrifft, erfolgt die Reduktion nach der Gleichung

(45) $2 \mathrm{HSNO}_{5}+\mathrm{SO}_{2}+2 \mathrm{H}_{2} \mathrm{O}=3 \mathrm{H}_{2} \mathrm{SO}_{4}+2 \mathrm{NO}$; ist aber Nitrosylschwefelsäure im Uberschuß vorhanden, so entsteht nitrosylige Schwefelsäure nach (46) $2 \mathrm{HSNO}_{5}+\mathrm{SO}_{2}+\mathrm{H}_{2} \mathrm{O}=\mathrm{N}_{2} \mathrm{O}_{3}\left(\mathrm{SO}_{3} \mathrm{H}\right)_{2}$

$$
+\mathrm{H}_{2} \mathrm{SO}_{4} \text {. }
$$

Wenn die Reduktion der Nitrosylschwefelsäure gemäß (45) bei Gegenwart von Kupfersulfat erfolgt, so bildet das freiwerdende Stickstoffoxyd mit Schwefelsäure und Kupfersulfat nitrosyligschwefelsaures Kupfer, und so ist es jedenfalls zu erklären, da $\beta$ bei der Einwirkung von überschüssigem Schwefeldioxyd auf nitrose Säure eine Bildung von nitrosyliger Schwefelsäure nur dann erfolgt, wenn Kupfersulfat vorhanden ist.

Die im letzteren Falle gebildete nitrosylige Schwefelsäure wird, wie die Versuche gezeigt haben, durch Schwefeldioxyd weiter reduziert. Ich habe Grund, anzunehmen, daß bei der Reduktion Stickstoffoxydul entsteht; hierbei ist der Reaktionsverlauf nach meiner Ansicht folgender:

$$
\begin{gathered}
\mathrm{O}_{2} \mathrm{~S}\left\langle\begin{array}{l}
\mathrm{OH} \\
\mathrm{O}-\mathrm{N}-\mathrm{N}-\mathrm{O}
\end{array} \mathrm{SO}^{\mathrm{HO}}+\mathrm{SO}_{2}+2 \mathrm{H}_{2} \mathrm{O}\right. \\
=3 \mathrm{H}_{2} \mathrm{SO}_{4}+\mathrm{N}=\mathrm{N}
\end{gathered}
$$

Falls beim Bleikammerprozeß nitrosylige Schwe- felsäure als Zwischenstufe auftreten sollte, müßten durch Reduktion derselben $\mathrm{zu} \mathrm{N}_{2} \mathrm{O}$ große Verluste an Salpeter entstehen. Da Stickstoffoxydul nicht oder nur in kleinen Mengen beim Bleikammerprozeß auftritt, so kann die nitrosylige Schwefelsäure dort als Zwischenstufe nicht in Frage kommen.

Die Ergebnisse meiner Versuche zusammenfassend, komme ich zu folgenden Schlüssen:

1. Die von Ras ch i g und L u nge-Berl für die Nitrosisulfosäure (Sulfonitronsäure) gegebenen Konstitutionsformel ist unwahrscheinlich, weil die angenommene Bildungsweise des Kupfersalzes z. B. aus $\mathrm{NO}, \mathrm{H}_{2} \mathrm{SO}_{4}$ und $\mathrm{CuSO}_{4}$ mit der Affinitätslehre unvereinbar ist.

2. Unter den in der Bleikammer bestehenden Bedingungen kann Nitrosisulfosäure (Sulfonitron. säure) gemäB der Theorie von $R$ a s chig, von $\mathrm{L} u \mathrm{ng}$ e und B e r l nicht entstehen.

3. Freie nitrosylige Schwefelsäure (Nitrosisulfosäure, Sulfonitronsäure) entsteht beim Bleikammerprozeß lediglich bei der Einwirkung von Schwefeldioxyd auf überschüssige Nitrosylschwefelsäure, z. B. bei fehlerhaftem Betrieb im Gay-Lussacturme. Für die Gewinnung der Schwefelsäure ist die Bildung der nitrosyligen Schwefelsäure belanglos oder schädlich.

4. Die Bleikammerkrystalle entstehen in der Kammer bei Wassermangel sekundär durch Einwirkung von nitrosen Gasen auf Schwefelsäure. Die Annahme einer primären Bildung von Nitrosylschwefelsäure durch Kondensation von $\mathrm{SO}_{2}, \mathrm{NO}, \mathrm{O}$ und $\mathrm{H}_{2} \mathrm{O}$ kann daher durch das Auftreten von Bleikammerkrystallen in der Kammer nicht begründet werden.

5. Die Annahme einer Zwischenstufe beim Bleikammerprozeß ist entbehrlich, weil die Oxydation des Schwefeldioxyds durch die nitrosen Gase unmittelbar erfolgt.

In den verschiedenen Theorien des Bleikammerprozesses tritt teils $\mathrm{N}_{2} \mathrm{O}_{3}$, teils $\mathrm{N}_{2} \mathrm{O}_{4}$ als oxydierendes Agens auf. Durch die Untersuchungen von $\mathrm{R}$ a $\mathrm{m}$ say, Condall, Lunge und Porschnew ist festgestellt, daB $\mathrm{N}_{2} \mathrm{O}_{3}$ im Gaszustande nicht existiert, und ist dieser Körper somit auch in der Bleikammer nicht enthalten. Dort kann die Oxydation des Schwefeldioxyds also nur durch Stickstoffdioxyd erfolgen, und kommen für die Schwefelsäurebildung in der Bleikammer nur folgende Reaktionen in Betracht: -

$$
\begin{aligned}
& \mathrm{SO}_{2}+\mathrm{NO}_{2}+\mathrm{H}_{2} \mathrm{O}=\mathrm{H}_{2} \mathrm{SO}_{4}+\mathrm{NO} \\
& \mathrm{NO}+\mathrm{O}=\mathrm{NO}_{2} .
\end{aligned}
$$

Auch im Gloverturm spielen die beiden Reaktionen eine große Rolle, es finden jedoch hier auch noch die durch die nachstehenden Gleichungen veranschaulichten Reaktionen statt:

(50) $2 \mathrm{HNO}_{2}+\mathrm{SO}_{2}=\mathrm{H}_{2} \mathrm{SO}_{4}+2 \mathrm{NO}$

(5I) $2 \mathrm{HSNO}_{5}+\mathrm{SO}_{2}+2 \mathrm{H}_{2} \mathrm{O}=3 \mathrm{H}_{2} \mathrm{SO}_{4}+2 \mathrm{NO}$.

Neben den eben angeführten Reaktionen treten beim Bleikammerprozeß noch Nebenreaktionen auf, die z. B. zur Bildung von Nitrosylschwefelsäure, nitrosyliger Schwefelsäure, zum Stickstoffoxydul und Ammoniak führen. [A. 141.] 